anni senza insorgenza di ceppi resistenti.L'incremento, dunque, di pazienti sottoposti a lunghi periodi di monitoraggio $\mathrm{HBV}$, renderebbe necessaria la disponibilità di test di facile esecuzione e poco costosi da utilizzare in supporto alle tecniche di biologia molecolare. Il metodo più semplice e meno costoso per diagnosticare l'infezione da HBV è la ricerca dell'HBsAg e dell'HBeAg nel siero del paziente con metodiche automatizzate (EIA, MEIA CMIA).

Metodi. Lo scopo di questo studio è di valutare la possibilità di monitorare pazienti affetti da epatopatia cronica B in terapia con lamivudina o adefovir mediante il dosaggio quantitativo di HBsAg CMIA (Architect, Abbott) da affiancare all'HBV DNA eseguito con bDNA (Versant HBV, Bayer).Il test $\mathrm{HBsAg}$ Architect è in grado di leggere un ampio range dinamico compreso tra $0.05 \mathrm{UI} / \mathrm{ml}$ e $250 \mathrm{UI} / \mathrm{ml}$; qualora $\mathrm{i}$ valori riscontrati eccedessero il limite superiore si è provveduto a una diluizione 1:100.

Risultati. Per studiare la concentrazione di $\mathrm{HBsAg}$ in relazione ad altri marker $\mathrm{HBV}$, si sono valutati 100 sieri $\mathrm{HBeAg}$ positivi e 100 sieri Anti HBe positivi, riscontrando una concentrazione significativamente superiore nei sieri HBeAg positivi $(\mathrm{p}<0.001)$. Si sono poi valutati 342 sieri raccolti da 78 pazienti $\mathrm{HBeAg}$ negativi a partire dal momento basale prima dell'inizio della terapia fino alla negativizzazione dell'HBV DNA, per evidenziare la concentrazione di HBsAg nel tempo, ma tale aspetto richiede una ulteriore valutazione con ampliamento della casistica.

Conclusioni. L'elaborazione dei dati sinora ottenuti non ci consente di estrapolare una valutazione univoca circa la possibilità di utilizzare l'attuale test HBsAg Architect quantitativo nei pazienti cronici, tenendo conto anche dell'attuale assoluto risalto che il test da alla sensibilità.

\title{
ANALISI QUANTITATIVA DI HBSAG MEDIANTE TECNICA CMIA IN PAZIENTI EPATOPATICI CRONICI
}

\author{
Lunghi G., Mascheroni E., Orlandi A., Spolti M., \\ Rampoldi S., *Lampertico P., *Viganò M., Pagano A. \\ Laboratorio Analisi Chimico Cliniche e Microbiologia, \\ *Dipartimento di Gastroenterologia - Ospedale Maggiore \\ Policlinico, Mangiagalli, Regina Elena, Fondazione IRCCS, Milano
}

Introduzione. Il virus HBV è la maggior causa di epatite cronica, cirrosi e carcinoma epatocellulare. La lamivudina è un analogo nucleosidico, in grado di sopprimere la replicazione virale e la progressione della malattia epatica. Tuttavia trattamenti prolungati possono indurre l'emergenza di varianti con mutazioni nel motivo tirosina-metioninaaspartato-aspartato del gene HBV polimerasi. Questi mutanti sono resistenti alla lamivudina e possono causare ricorrenze virologiche e/o biochimiche durante la terapia. E' estremamente importante che durante la terapia sia mantenuto costante il monitoraggio del livello di HBV DNA e $\mathrm{HBeAg}$ per evidenziare precocemente la presenza di ceppi resistenti.L'utilizzo di un nuovo farmaco, l'Adefovir, consente inoltre di controllare la replicazione di HBV per lunghi 